\title{
SERGENTOMYIA BERNARDAE N. SP. (DIPTERA, PSYCHODIDAE)
}

\section{Espèce nouvelle récoltée dans le Mayombe congolais}

\author{
J. TROUILLET*
}

(Collaboration technique : A. S. Bimangou et J. M. Boudzoumou)

RÉSUMÉ. Sergentomyia bernardae, nouvelle espèce récoltée dans le massif forestier du Mayombe en République Populaire du Congo, est décrit d'après 4 exemplaires mâles et 28 exemplaires femelles. Ce nouveau Phlébotome, morphologiquement proche de $S$. hamoni, S. dissimillima, $S$, simillima, se distingue de la première espèce par l'absence d'épine géniculée sur le $3^{\mathrm{e}}$ segment de l'antenne et des deux autres par l'armature cibariale.

\section{Sergentomyia bernardae n. sp. (Diptera, Psychodidae), a new species from the congolense Mayombe}

SUMMARY. Sergentomyia bernardae, new species collected in the Mayombe forest in the People's Republic of Congo, is described from 4 male specimens and 28 female specimens. This sandfly is differenciated from $S$. hamoni by the lack of the geniculated spine on the antennal segment III and is differenciated from $S$. dissimillima and $S$. simillima by the cibarial armature.

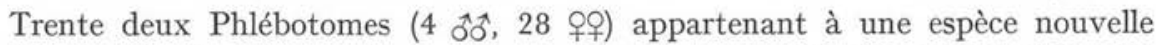
ont été récoltés dans le massif forestier du Mayombe en République Populaire du Congo (Vattier-Bernard et Trouillet, 1978) ${ }^{1}$. Un seul individu a été capturé à la main, en forêt, sur tronc d'arbre ; les autres ont été pris dans quatre stations différentes à l'aide d'un piège adhésif lumineux (Rioux et coll., 1969) installé en lisière de forêt (tableau I).

Cette espèce est dédiée à Madame le Professeur G. Vattier-Bernard en témoignage de reconnaissance et de respectueuse amitié.

* Département de Biologie animale, Equipe de Bio-écologie des Vecteurs, Faculté des Sciences, BP 69, Brazzaville, République Populaire du Congo.

I. Travail effectué dans le cadre de l'Équipe pluridisciplinaire de Biologie forestiére tropicale de Dimonika.

Accepté le 5 décembre $198 \mathrm{r}$. 
Après S. bergerardi Trouillet et Vattier-Bernard, 1978, S. roberti Vattier-Bernard et Trouillet, 1981 S. horridula Vattier-Bernard et Trouillet, 1982 S. bernardae est la quatrième espèce nouvelle trouvée dans la partie congolaise du massif du Mayombe.

Tableau I. - Stations de récolte de $S$. bernardae.

\begin{tabular}{|c|c|c|c|c|c|}
\hline \multicolumn{2}{|c|}{ Stations } & \multicolumn{2}{|r|}{ Date } & \multirow{2}{*}{$\begin{array}{c}\begin{array}{c}S . \text { ber- } \\
\text { nardae } \\
\text { capturés }\end{array} \\
1 \text { 우 }\end{array}$} & \multirow{2}{*}{$\begin{array}{c}\text { Mode de } \\
\text { piègeage }\end{array}$} \\
\hline $\begin{array}{l}\text { Dimonika (0414'S - } \\
\left.12^{\circ} 26^{\prime} \mathrm{E}\right)\end{array}$ & $\begin{array}{l}\text { Case en bois de } \\
\text { l'Université }\end{array}$ & Nuit & du 15 au 16.3 .1977 & & \\
\hline $\begin{array}{l}\text { Dimonika (04\%14'S - } \\
\left.\quad 12^{\circ} 26^{\prime} \mathrm{E}\right)\end{array}$ & $\begin{array}{l}\text { Ancienne Case } \\
\text { François }\end{array}$ & $\begin{array}{c}\text { Nuit } \\
" \\
" \\
" \\
" \\
" \\
" \\
" \\
"\end{array}$ & $\begin{array}{l}\text { du } 24 \text { au } 25.3 .1977 \\
\text { du } 16 \text { au } 17.1 .1978 \\
\text { du } 14 \text { au } 15.11 .1980 \\
\text { du } 23 \text { au } 24.01 .1981 \\
\text { du } 20 \text { au } 21.02 .1981 \\
\text { du } 07 \text { au } 08.03 .1981 \\
\text { du } 09 \text { au } 10.03 .1981 \\
\text { du } 15 \text { au } 16.04 .1981 \\
\text { du } 25 \text { au } 26.04 .1981\end{array}$ & $\begin{array}{ll}1 & \text { + } \\
1 & 0 \\
1 & 0 \\
1 & 0 \\
1 & 0 \\
1 & + \\
1 & \text { q } \\
1 & 0 \\
1 & \text { q } \\
1 & +\end{array}$ & $\begin{array}{c}\text { PAL } \\
" \\
" \\
" \\
" \\
" \\
" \\
" \\
"\end{array}$ \\
\hline $\begin{array}{l}\text { Makaba }\left(04^{\circ} 09^{\prime} \mathrm{S}-\right. \\
\left.12^{\circ} 24^{\prime} \mathrm{E}\right)\end{array}$ & Case Gaston & $\begin{array}{c}\text { Nuit } \\
" \\
" \\
\text { Semaine } \\
" \\
" \\
" \\
" \\
" \\
" \\
" \\
" \\
"\end{array}$ & $\begin{array}{l}\text { du } 21 \text { au } 22.03 .1978 \\
\text { du } 22 \text { au } 23.03 .1978 \\
\text { du } 26 \text { au } 27.03 .1978 \\
\text { du } 28 \text { au } 29.03 .1978 \\
\text { du } 15 \text { au } 21.05 .1978 \\
\text { du } 26.6 \text { au } 02.07 .1978 \\
\text { du } 03 \text { au } 09.07 .1978 \\
\text { du } 10 \text { au } 16.07 .1978 \\
\text { du } 07 \text { au } 13.08 .1978 \\
\text { du } 30.10 \text { au } 05.11 .1978 \\
\text { du } 06 \text { au } 12.11 .1978 \\
\text { du } 04 \text { au } 10.12 .1978 \\
\text { du } 11 \text { au } 17.12 .1978 \\
\text { du } 25 \text { au } 31.12 .1978\end{array}$ & 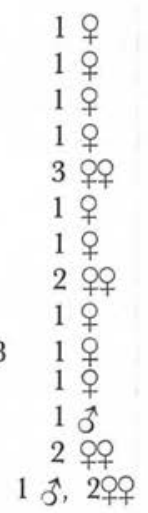 & $\begin{array}{c}\text { PAL } \\
" \\
" \\
" \\
" \\
" \\
" \\
" \\
" \\
" \\
" \\
"\end{array}$ \\
\hline $\begin{array}{l}\text { Pounga (04. } 16^{\prime} \mathrm{S}- \\
\left.12^{\circ} 27^{\prime} \mathrm{E}\right)\end{array}$ & Case Boungou & Nuit & du 21 au 22.03.1978 & 1 우 & PAL \\
\hline $\begin{array}{l}\text { Forêt entourant la ba } \\
\text { de Faustin (bas), entr } \\
\text { de Kouilila (04.11's }\end{array}$ & $\begin{array}{l}\text { ananeraie } \\
\text { rée sud } \\
\left.-12^{\circ} 26^{\prime} \mathrm{E}\right)\end{array}$ & & 14.07 .1978 & 19 & $\mathrm{CM}$ \\
\hline
\end{tabular}

* PAL : Piège Adhésif Lumineux ; CM : Capture Manuelle. 


\section{Description}

Femelle : La description est faite à partir de l'holotype no $30584 \mathrm{JT}$ et des paratypes $\mathrm{n}^{\circ} 31315 \mathrm{JT}, \mathrm{n}^{\circ} 35721 \mathrm{JT}, \mathrm{n}^{0} 35754 \mathrm{JT}, \mathrm{n}^{\circ} 35825 \mathrm{JT}, \mathrm{n}^{\circ} 35857 \mathrm{JT}, \mathrm{n}^{\circ} 35910 \mathrm{JT}$, $\mathrm{n}^{\circ} 36666 \mathrm{JT}, \mathrm{n}^{\circ} 36667 \mathrm{JT}, \mathrm{n}^{\circ} 36668 \mathrm{JT}, \mathrm{n}^{\circ} 37329 \mathrm{JT}, \mathrm{n}^{\circ} 37418 \mathrm{JT}, \mathrm{n}^{\circ} 38244 \mathrm{JT}$, $\mathrm{n}^{\circ} 38712 \mathrm{JT}, \mathrm{n}^{\circ} 38713 \mathrm{JT}, \mathrm{n}^{\circ} 38857 \mathrm{JT}$, no $39653 \mathrm{JT}, \mathrm{n}^{\circ} 39712 \mathrm{JT}, \mathrm{n}^{\circ} 39900 \mathrm{JT}$, no 39901 JT, no 40589 JT, no 40590 JT, no 44633 GVB-JT, no 45372 GVB-JT, no 45415 GVB-JT no 45420 GVB-JT, no 45608 GVB-JT, no 45653 GVB-JT. Les mesures ont été effectuées sur les Insectes éclaircis à la potasse à $20 \%$, mordancés dans le liquide de Berlèse, déshydratés et montés dans le baume du Canada.

Taille: $2,21 \mathrm{~mm}(1,97-2,30-2,71)^{1}$.

Patte postérieure : longueur totale : $3,30 \mathrm{~mm}(3,24-3,35-3,46)$; fémur : $0,82 \mathrm{~mm}$ $(0,80-0,83-0,85)$; tibia : $1,21 \mathrm{~mm}(1,17-1,23-1,31)$; tarse : $1,27 \mathrm{~mm}(1,27-$ $1,28-1,31)$.

Aile $(P l . I, A)$ : longueur : $2,03 \mathrm{~mm}(1,95-2,07-2,28)$; largeur : $0,58 \mathrm{~mm}(0,55-$ $0,62-0,68)$; rapport longueur/largeur : $3,50(3,17-3,35-3,55)$; alpha : $0,50 \mathrm{~mm}$ $(0,41-0,48-0,56)$; bêta : $0,34 \mathrm{~mm}(0,33-0,36-0,42)$; rapport alpha/bêta : 1,50 $(1,02-1,35-1,75)$; delta : $+0,30 \mathrm{~mm}(0,19-0,29-0,35)$; gamma : $+0,23 \mathrm{~mm}$ $(0,23-0,27-0,33) ; \mathrm{pi}:+0,10 \mathrm{~mm}(0,07-0,12-0,16)$.

Labre-épipharynx : longueur : $0,18 \mathrm{~mm}(0,17-0,18-0,19)$.

Antenne (Pl. I, B, C) : longueur du segment III : 0,33 mm $(0,33-0,35-0,38)$; longueur du segment IV : $0,16 \mathrm{~mm}(0,16-0,17-0,19)$; longueur du segment $\mathrm{V}$ : $0,17 \mathrm{~mm}(0,16-0,17-0,19)$; rapport $\mathrm{A}$ III/E : $1,83(1,79-1,95-2,12) ; \mathrm{III}=$ $\mathrm{IV}+\mathrm{V} ; \mathrm{IV}=\mathrm{V}$; formule antennaire $: 2 / \mathrm{IV}-\mathrm{XV}$.

Palpe : longueur totale : $0,69 \mathrm{~mm}(0,61-0,67-0,72)$; formule : $1-2-3-4-5$. Cibarium (Pl. I, D, E) : armé de 7 à 9 dents bien individualisées, triangulaires, acérées, à arête centrale longitudinale. Ni denticule, ni tache pigmentée observables. Cette armature cibariale présente une évidente fragilité, les dents sont fréquemment détériorées.

Pharynx (Pl. I, D) : 0,17 $\mathrm{mm}$ de long sur $0,14 \mathrm{~mm}$ dans sa plus grande largeur. Typiquement cordiforme dans sa partie postérieure, il est armé d'un grand nombre de denticules prolongés par une épine à extrémité bifide.

Spermathèques $(P l . I, F)$ : à parois lisses. Les dimensions moyennes sont $40 \mu \mathrm{m}$ de longueur sur $18 \mu \mathrm{m}$ de largeur.

Mâle: La description est faite à partir de l'allotype no $27530 \mathrm{GVB}$ et des paratypes n 39846 JT, n 40616 JT, no 45282 GVB-JT.

I. La dimension de l'holotype est suivie, entre parenthèses, de la moyenne encadrée par les extrêmes mesurés. 

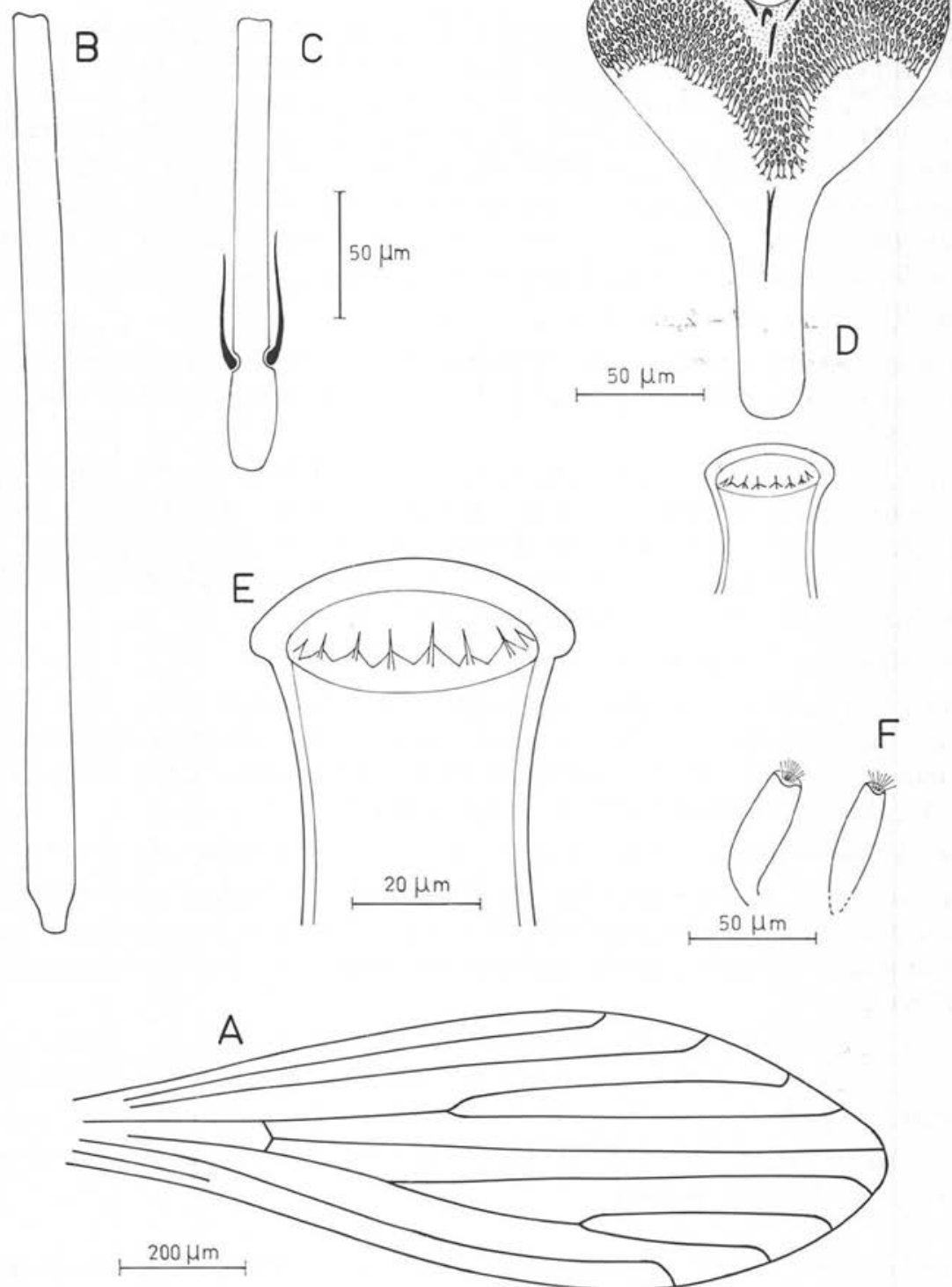

Planche I. - Sergentomyia bernardae femelle: A, aile; B et C, segments III et IV de l'antenne $\mathrm{D}$, cibarium et pharynx; $\mathrm{E}$, cibarium vu à l'immersion; $\mathrm{F}$, spermathèques. 

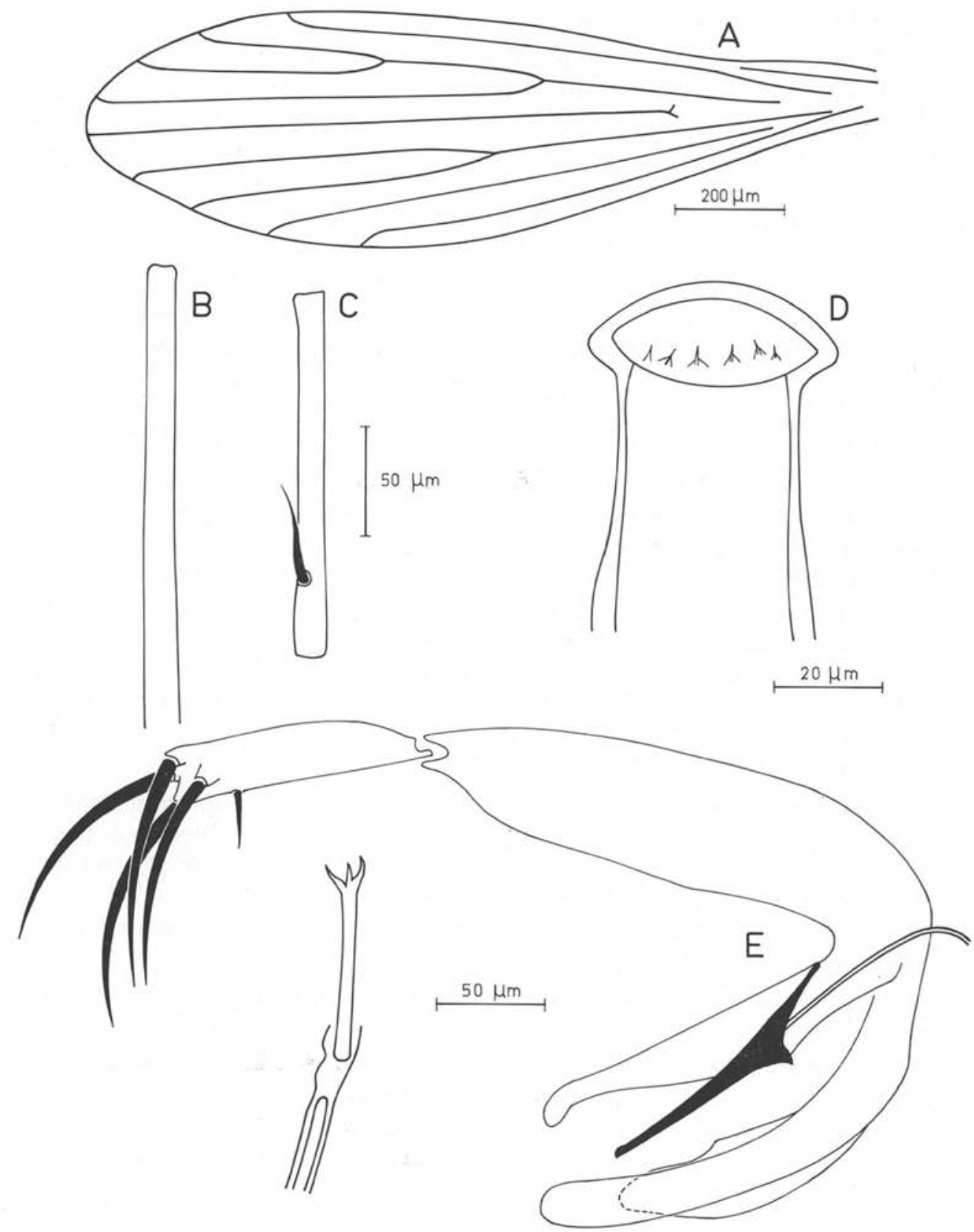

Planche II. - Sergentomyia bernardae mâle : A, aile; B et C, segments III et IV de l'antenne; $\mathrm{D}$, cibarium vu à l'immersion; $\mathrm{E}$, génitalia et pompe génitale. 
Taille: $2,28 \mathrm{~mm}(2,43-2,39-2,16)^{1}$.

Patte postérieure: longueur totale : NM $(\mathrm{NM}-\mathrm{NM}-2,89 \mathrm{~mm})^{2}$; fémur : NM (NM $\mathrm{NM}-0,73 \mathrm{~mm})$; tibia : NM $(\mathrm{NM}-\mathrm{NM}-1,06 \mathrm{~mm})$; tarse : NM $(\mathrm{NM}-\mathrm{NM}-1,10 \mathrm{~mm})$. Aile $(P l . I I, A)$ : longueur : $1,70 \mathrm{~mm}(1,56-1,68-1,65)$; largeur : $0,42 \mathrm{~mm}(0,42-$ $0,44-0,45)$; rapport longueur/largeur : $4,05(3,71-3,82-3,67)$; alpha : $0,33 \mathrm{~mm}$ $(0,26-0,28-0,34)$; bêta : $0,30 \mathrm{~mm}(0,31-0,32-0,29)$; rapport alpha/bêta : 1,10 $(0,84-0,88-1,17)$; delta $:+0,16(0,10-0,11-0,17)$; gamma : $+0,23 \mathrm{~mm}(0,26-$ $0,27-0,23) ; \mathrm{pi}:+0,10(0,11-0,09-0,12)$.

Labre-épipharynx : longueur : $0,16 \mathrm{~mm}(0,14-0,16-0,15)$.

Antenne (Pl. II, B, C) : longueur du segment III : 0,36 mm $(0,34-0,39-0,36)$; longueur du segment IV : $0,18 \mathrm{~mm}(0,18-0,20-0,18)$; longueur du segment $\mathrm{V}$ : $0,19 \mathrm{~mm}(0,18-0,20-0,18) ;$ rapport A III/E : $2,25(2,43-2,44-2,40) ; \mathrm{III}=\mathrm{IV}+$ $\mathrm{V} ; \mathrm{IV}=\mathrm{V} ;$ formule antennaire $: 1 / \mathrm{IV}-\mathrm{XV}$.

Palpe : longueur totale : NM (NM - 0,66 mm - NM) ; formule : 1-2-(3-4)-5.

Cibarium $(P l . I I, D)$ : armé de 6 à 7 dents très comparables à celles de la femelle mais de taille plus réduite. Ni denticule, ni tache pigmentée observables.

Pharynx : 0,14 mm de long sur 0,04 $\mathrm{mm}$ de large ; il présente quelques replis postérieurs finement denticulés.

Génitalia ( $P l . I I, E)$ : le style porte 4 épines terminales et 1 soie interne, le fourreau pénien est en forme de cône allongé à extrémité mousse. Coxite : $0,24 \mathrm{~mm}(0,24-$ $0,24-\mathrm{NM})$; style : $0,12 \mathrm{~mm}(0,11-0,11-0,11)$; paramère : $0,18 \mathrm{~mm}(0,18-0,18$ $0,18)$; lobe latéral : $0,19 \mathrm{~mm}(0,17-0,19-0,18)$; fourreau pénien : $0,12 \mathrm{~mm}(0,13-$ $0,11-0,11)$; filaments génitaux : $0,28 \mathrm{~mm}(0,25-0,28-0,24)$; pompe génitale : $0,11 \mathrm{~mm}(0,09-0,10-0,09)$; rapport FG/PG : 2, $5(2,8-2,8-2,7)$.

Cette espèce apparaît morphologiquement proche de S. hamoni (Abonnenc, 1958), S. dissimillima (Abonnenc, 1972) et S. simillima (Newstead, 1914). L'absence d'épine géniculée sur le $3^{\mathrm{e}}$ segment de l'antenne la distingue de $S$. hamoni, tandis que l'armature cibariale la différencie nettement des deux autres espèces :

- S. dissimillima présente un cibarium muni de dents courtes, réfringentes et difficilement visibles ;

- S. simillima est armé de dents subégales disposées en ligne presque droite (" en palissade ");

- S. bernardae possède des dents triangulaires, acérées, bien individualisées, rangées suivant une courbe régulière à concavité postérieure.

Les essais de détermination de cette espèce avec la clé de détermination d'Abonnenc (1972) conduisent à $S$. simillima pour le mâle et pour la femelle à l'impasse ci-après :

I. La dimension de l'allotype est suivie, entre parenthèses, de celles des paratypes.

2. $\mathrm{NM}=$ appendice ou article non mesuré car déformé ou absent. 
88 - Cibarium armé de 18-30 dents disposées en arc à concavité postérieure; les dents latérales sont plus fortes que les médianes; segment III de l'antenne

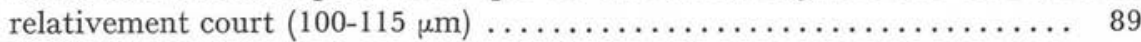

- Cibarium armé de 10 à 15 dents subégales disposées en ligne presque droite ; segment III de l'antenne relativement long $\ldots \ldots \ldots \ldots \ldots \ldots \ldots \ldots \ldots .90$

Il est possible d'introduire $S$. bernardae dans cette clé de la façon suivante

Mâle

$44-3^{e}$ segment de l'antenne dépourvu d'épine géniculée ; 10-12 dents au cibarium ............................ simillima (Newstead, 1914).

- 3e segment de l'antenne dépourvu d'épine géniculée ; 6 à 7 dents acérées, bien

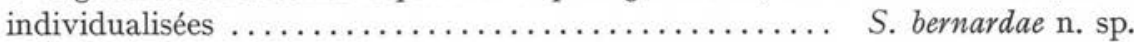

- 3e segment de l'antenne portant une épine géniculée bien développée .... 45

Femelle

88 - Cibarium armé de 18-30 dents disposées en arc à concavité postérieure ; les dents latérales sont plus fortes que les médianes; segment III de l'antenne

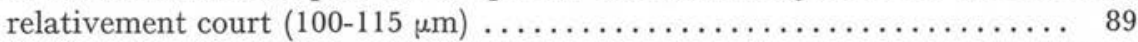

- Cibarium armé de 10 à 15 dents subégales disposées en ligne presque droite ; segment III de l'antenne relativement long $\ldots \ldots \ldots \ldots \ldots \ldots \ldots \ldots \ldots .90$

- Cibarium armé de 7 à 9 dents bien individualisées, triangulaires, acérées, munies d'une arête centrale longitudinale; segment III de l'antenne mesurant environ $350 \mu \mathrm{m} \ldots \ldots \ldots \ldots \ldots \ldots \ldots \ldots \ldots \ldots \ldots \ldots \ldots \ldots \ldots \ldots \ldots \ldots \ldots$ S. bernardae $\mathrm{n} . \mathrm{sp}$.

Dépôt des types: L'holotype no 30584 JT et l'allotype no 27530 GVB seront déposés au Muséum d'Histoire Naturelle de Paris.

\section{BIBLIOGRAPHIE}

Abonnenc E. : Sur Phlebotomus simillimus Newstead, 1914 et ses variétés. Arch. Inst. Pasteur Algér., 1958, 37, 591-593.

Abonnenc E. : Les Phlébotomes de la région éthiopienne (Diptera, Psychodidae). Mém. O.R.S.T.O.M., 1972, 55,289 p.

Newstead R. : Notes on Phlebotomus, with description of new species. Part. III. Bull. ent. Res., I9I4, S, I79-I92.

Rioux J. A., Golvan Y. J., Croset H., Tour S., Houin R., Abonnenc E., Petitdidier M., Vollhardt Y., Dedet J. P., Albaret J. L., Lanotte G., Quilici M. : Épidémiologie des Leishmanioses dans le Sud de la France. Monographie INSERM, I969, 37, $221 \mathrm{p}$.

Trouillet J., VAtTIER-Bernard G. : Sergentomyia (Sergentomyia) bergerardi n. sp. (Diptera, Phlebotomidae). Espèce nouvelle récoltée en République Populaire du Congo. Ann. Parasitol. Hum. Comp., 1978, 53, 705-709.

VAttier-Bernard G., Trouillet J. : Les Phlébotomes (Diptera, Phlebotomidae) du Mayombe (République Populaire du Congo). Ann. Parasitol. Hum. Comp., 1978, 53, 697-704.

Vattier-Bernard G., Trouillet J. : Sergentomyia roberti (Diptera, Psychodidae). Espèce nouvelle récoltée au Congo. Ann. Parasitol. Hum. Comp., I981, s6, 54 I-545.

Vattier-Bernard G., Trouillet J. : Sergentomyia horridula (Diptera, Psychodidae). Nouveau Phlébotome récolté dans le Mayombe (Congo). Ann. Parasitol. Hum. Comp., 1982, 57, $301-306$. 